\title{
Percepción social de riesgo: una mirada general y aplicación a la comunicación de salud
}

\author{
Milena Stanojlovic \\ Doctora por la Universidad Complutense de Madrid
}

\section{Palabras clave}

Percepción de riesgo Paradigma psicométrico Comunicación de riesgos en salud

\begin{abstract}
Resumen
La percepción de riesgo es el componente central en los modelos teóricos de comportamiento y la toma de decisiones. El presente artículo ofrece una revisión del estado actual de la investigación de la percepción social de riesgo. Especialmente, nos enfocaremos en la percepción de riesgos en salud y la tendencia de los individuos a menospreciar el riesgo personal de determinada enfermedad. Conocer la naturaleza de la percepción de riesgo personal de los individuos es clave para el desarrollo de la comunicación en salud más efectiva.
\end{abstract}




\section{PUBLIC PERCEPTION OF RISK: A GENERAL PERSPECTIVE AND APPLICATION TO HEALTH COMMUNICATION}

\section{Keywords}

Risk perception psychometric paradigm health risk communication

\begin{abstract}
Risk perception is a central component in behavioral and decision making theories. The present article aims to review the present state of knowledge in public perception of risk. More specifically, we will focus on health risk perception and the people's tendency to underestimate the probability of personal health risk. Understanding the nature of risk perception is key for developing more effective health risk communication.
\end{abstract}

\section{Cómo citar el artículo}

Stajnolovic, M. (2015). Percepción social de riesgo: una mirada general y aplicación a la comunicación de salud. Revista de Comunicación y Salud, Vol. 5, pp. 99-110.

DOI: http://doi.org/10.35669/revistadecomunicacionysalud.2015.5.99-110 


\section{Percepción de riesgo en la sociedad}

La investigación sobre la percepción social de riesgo tuvo sus inicios en los estudios del razonamiento y la toma de decisiones, en el ámbito progresivo del análisis de riesgo en las sociedades industrializadas. El análisis de riesgo, además de su aspecto científico reflejado en la evaluación y gestión de riesgo, tiene un marcado aspecto político, que abarca la percepción social de riesgo, valores, procedimiento, poder, confianza y polémicas sociales (ver la representación esquemática en Slovic y Weber, 2002: 3).

Con el objetivo de mejorar la comunicación de riesgo entre las instituciones responsables y la población, se pretendía (1) descubrir qué era lo que los ciudadanos consideraban como riesgo y por qué, (2) desarrollar una teoría de percepción de riesgo mediante la cual se pudieran predecir sus respuestas frente a nuevos riesgos y (3) desarrollar las técnicas de evaluación de las opiniones complejas y sutiles que las personas tienen sobre el riesgo (Slovic, Fischhoff, y Lichtenstein, 1982). La percepción social de riesgo, formada bajo la luz de diferentes factores, tiene un papel crucial en el análisis de riesgo porque condiciona el proceso de la toma de decisiones de los individuos al enfrentarse con las situaciones arriesgadas. En otras palabras, la respuesta protectora del individuo está directamente relacionada con sus percepciones de riesgo inmediatamente anteriores a la acción realizada.

Según la observación de Chauvin, Hermand, y Mullet (2008), la mayoría de la investigación sobre percepción de riesgo se ha centrado en dos preguntas: 1. "¿Por qué ciertos riesgos se perciben como más peligrosos que otros por la población general?" y 2. “¿Por qué los individuos se diferencian en su percepción del riesgo asociado a un mismo peligro?"

Tratando de contestar a la primera pregunta, la percepción de riesgo se ha abordado desde tres paradigmas diferentes: el paradigma de medición axiomática, el socio-cultural y el psicométrico (para la revisión consultar Weber, 2001). El primero de ellos, el paradigma de medición axiomática, estudia la manera de los individuos de transformar la información objetiva o científica en conclusiones subjetivas sobre el impacto que ciertos acontecimientos de riesgo pueden tener sobre su vida. El paradigma socio-cultural estudia la influencia del entorno social en la percepción de riesgo. Finalmente, el paradigma psicométrico, la corriente más prolífica fundamentada en la psicología cognitiva, tiene como idea central un conjunto mental de heurísticos o factores emocionales que condicionan la percepción de riesgo y guían la toma de decisiones humana con la intención de racionalizar o entender un futuro incierto (Fischhoff, Slovic, Lichtenstein, Read, y Combs, 1978; Slovic, 1987). En concreto, estos estudios empíricos demostraron que los sentimientos de terror eran el mayor determinante de la percepción y la aceptación pública de riesgo para diferentes tipos de peligro (Slovic, Finucane, Peters, y MacGregor, 2004: 314). En el marco de este paradigma, se empezaron a utilizar las representaciones cuantitativas de las actitudes y percepciones de riesgo, demostrando que la percepción de riesgo 
es "previsible y cuantificable" (Slovic y Weber, 2002: 7). Fischhoff et al. (1978) desarrollaron la clasificación taxonómica de posibles peligros y mediante la medición psicométrica identificaron los factores principales que condicionan la percepción de riesgo:

(1) el nivel de conocimiento sobre el riesgo,

(2) el nivel de terror que el riesgo induce entre los individuos y

(3) el número de individuos expuestos a él.

De acuerdo con las teorías duales de procesamiento de la información (Epstein, 1994; Sloman, 1996), el riesgo se percibe, evalúa y controla de dos maneras fundamentales: por un lado, riesgo como análisis objetivo (risk as analysis), que incluye lógica, razón y reflexión científica relacionada con la evaluación y la toma de decisiones, y por otro, riesgo como sentimiento (risk as feeling) referido a nuestras reacciones instintivas, intuitivas y automáticas al peligro (Slovic, y Peters, 2006: 322; Slovic et al., 2004).

Por tanto, la percepción social de riesgo no se puede reducir a una simple relación de probabilidades y previsión de consecuencias, sino que depende de una serie de factores cualitativos como creencias, opinión pública y otros factores relacionados con los efectos indeseables que causan terror y que los expertos suelen ignorar. En este sentido, la percepción pública puede diferenciarse de la percepción científica de riesgo, porque no se basa solamente en datos objetivos. Más bien, los marcos de referencia para la percepción pública de riesgo suelen fundamentarse en criterios subjetivos o en fuentes no científicas y no siempre tan fiables, tales como las decisiones políticas, las creencias dominantes y la información proporcionada por los medios de comunicación, que en medida significativa condicionan la percepción real sobre el riesgo, incluso más que la evaluación científica basada en los estudios empíricos.

Muchas teorías consideran que el componente afectivo suele prevalecer sobre el componente racional en la motivación de comportamiento y la toma de decisiones (eg. Barrett y Salovey, 2002; Zajonc, 1980). En la vida cotidiana a la hora de juzgar y decidir sobre riesgo, solemos guiarnos más por los sentimientos intuitivos y automáticos (miedo, ira, ansiedad, asco) que por los criterios analíticos y racionales, aunque la vida esté en juego. Este método de apoyo en los sentimientos denominado heurístico del afecto (affect heuristic) es la manera más rápida, más fácil y más eficiente de navegar en un mundo incierto y a veces peligroso que medir todas las razones a favor y en contra, especialmente cuando el razonamiento es costoso y las capacidades son limitadas (Slovic, Peters, Finucane, y MacGregor, 2005: S35).

Otro cuerpo de estudios se ha centrado en los factores que condicionan que algunos individuos perciben riesgo asociado a un peligro diferente que otros individuos. La investigación ha mostrado que la percepción de riesgo varía en función de diferentes factores demográficos, socioeconómicos y culturales, así como 
en función de la experiencia previa con el peligro, el consumo de los medios de comunicación y la confianza en los organismos reguladores (para la revisión bibliográfica ver Brady, 2012: 548). Asimismo, algunos estudios examinaron los factores de personalidad, las creencias o la visión del mundo (Bouyer et al. 2001) como factores que condicionan la percepción de riesgo.

\section{Percepción de riesgo en la comunicación de salud}

Diferentes modelos teóricos de comportamiento en salud, como el Modelo de procesos paralelos extendido (Witte, 1992), la Teoría de la motivación de protección de Rogers $(1975,1983)$ o el Modelo de creencias de salud (Rosenstock, 1974) reconocen la percepción de riesgo como el componente fundamental y antecedente necesario (aunque no siempre suficiente) para el cambio de intención y comportamiento del receptor. Los meta-análisis han comprobado que, en general, la percepción de riesgo está positivamente asociada tanto con la intención (Floyd, Prentice-Dunn, y Rogers, 2000), como con el cambio del comportamiento de salud (Brewer et al., 2007).

De acuerdo con el paradigma psicométrico, las respuestas afectivas condicionan la percepción de riesgo también en el ámbito de salud. Si las emociones hacia cierta actividad, que además se realiza habitualmente, son favorables, los individuos tienden a juzgar los riesgos como bajos y los beneficios como altos; en el caso contrario, perciben los altos riesgos y bajos beneficios (Slovic et al., 2005). A modo de ejemplo, hace tiempo que se demostró que el grado de riesgo percibido por los jóvenes sobre el consumo de marihuana es inverso a los niveles de su consumo: cuanto menor sea la percepción de riesgo del individuo, la probabilidad de consumo es mayor, y viceversa (Bachman, Johnston, O’Malley, y Humphrey, 1988). En cambio, la imagen de cáncer como una enfermedad terrorífica y frecuentes historias de cáncer aumentan la percepción de riesgo, aunque la probabilidad objetiva personal de sufrir un cáncer es pequeña. No obstante, aunque el riesgo está reducido pero no eliminado, el miedo de cáncer puede mantenerse y continuar de condicionar altas percepciones de riesgo, a pesar de su verdadera reducción (Slovic et al., 2005: S38).

Los medios de comunicación juegan un papel importante en la formación de la percepción pública de riesgo en el ámbito de prevención y promoción de la salud. Las campañas de salud aspiran a incrementar tanto la notoriedad de cierto asunto como el reconocimiento del riesgo personal de las consecuencias negativas como resultado de un comportamiento nocivo, con el objetivo final de provocar un cambio de conducta.

La investigación reciente sobre la percepción de riesgo en salud puede ser dividida en dos grandes categorías: la primera en la que se examinan las percepciones de riesgo y la toma de decisiones que se refieren a los hábitos de riesgo realizados por el propio individuo tales como: fumar, beber, conducir en estado alcoholizado, protección solar, comportamiento adictivo etc.; y la segunda 
abarca la percepción de riesgo de todos los acontecimientos sobre los cuales el individuo tiene poco o ningún control, como catástrofes naturales o antrópicos (para la revisión bibliográfica ver Brady, 2012). Nuestro interés se enfoca en la primera categoría, en las conductas de riesgo realizadas como fruto de las decisiones y hábitos del propio individuo.

\subsection{Definición y medición de la percepción de riesgo en salud}

El constructor de la percepción de riesgo ha sido definido y evaluado de diferentes maneras a lo largo de la investigación y se ha entendido como "la probabilidad percibida", "vulnerabilidad percibida" o "la susceptibilidad". El metaanálisis de Brewer et al. (2007) aporta una mayor precisión terminológica y metodológica sobre las dimensiones de este constructo complejo reflejadas en el contexto de salud. Distingue claramente entre dos términos a menudo utilizados indistintamente:

1) la percepción individual de la probabilidad de daño (¿Cuál es la probabilidad de que obtengas gripe este año si no recibes la vacuna?) y

2) la percepción de la susceptibilidad general del individuo al daño, es decir, a la enfermedad (¿Te enfermas fácilmente de gripe? ¿ ¿Tienes más probabilidad que otras personas de enfermarte de gripe?).

La tercera dimensión inconfundible es:

3) la severidad percibida de las consecuencias (¿Cómo de severa para ti es esta enfermedad?).

Las conclusiones de este estudio revelan que las tres medidas de la percepción de riesgo y cada una por separado fueron positivamente asociadas con el comportamiento de obtener vacuna (Brewer et al., 2007). En otros estudios del campo, la primera dimensión descrita anteriormente y la más usada para evaluar la percepción de riesgo se denomina "la probabilidad absoluta de riesgo" numérica o verbal (absolute probability scales), mientras que la susceptibilidad percibida se denomina también "la percepción de riesgo comparada" con otros individuos promedios parecidos (comparative risk perception) (Dillard et al., 2012).

Junto con estas medidas tradicionales, en la bibliografía sobre la percepción de riesgo se destaca otra medida recién desarrollada: el sentimiento de riesgo (feelings of vulnerability) (Weinstein et al., 2007; Dillard et al., 2012). Este factor evalúa cuánto se siente vulnerable el sujeto si no lleva a cabo el comportamiento recomendado de prevención. En la línea de los ejemplos anteriores, se formularía una afirmación "Si no recibo la vacuna, me sentiría muy vulnerable a gripe". La percepción de riesgo expresada en términos de sentimiento en vez de en términos cognitivos de pura evaluación de probabilidad, se mostró como el mejor predicador del futuro comportamiento de vacunación (Weinstein et al., 2007), de la actitud favorable y del comportamiento real de screening de cáncer de colón (Dillard et al., 2012). 
Estas conclusiones del ámbito de salud son congruentes con el paradigma psicométrico de la teoría de riesgo que las personas sobre todo sentimos riesgo, y que el componente afectivo prevalece sobre el racional a la hora de percibir y controlar el riesgo de peligro.

Teniendo en cuenta de que estas mediciones pueden abarcar diferentes dimensiones del constructo general de la percepción de riesgo, Dillard et al. (2012) advierten que incluir solo una medición puede ser problemático y resultar en conclusiones erróneas y correlaciones con las intenciones y comportamiento de salud muy variadas (2012: 106). Asimismo, destacan la necesidad de que los investigadores evalúen la percepción de riesgo combinando diferentes medidas, el sentimiento de la vulnerabilidad junto con otras medidas más tradicionales. Otra observación a nivel metodológico, según Brewer et al. (2007), se refiere a la necesidad de resolver los problemas en los estudios empíricos debidos a falta de los matices sutiles entre estos conceptos, formulando las preguntas más rigurosas a los receptores. Una pregunta mal formulada puede no dar resultados fiables: "If the measure assesses something other than the intended likelihood or severity or is statistically unreliable, it is not surprising for it to be unrelated to vaccination" (Brewer et al., 2007: 142).

\subsection{Falta de la percepción de riesgo personal como resistencia a la persuasión}

A pesar de la potencial importancia de una correcta percepción de riesgo, los individuos tienden a interpretar su propio riesgo de la manera que mejor les conviene, de una manera auto-protectora (Morton y Duck, 2001: 604). En cambio, el riesgo que se le atribuye a otros es exagerado en comparación con la percepción de riesgo personal, reflejando el "sesgo optimista" (Weinstein, 1980). En ese contexto, Morton y Duck (2001) descubrieron que mientras que la información sobre el cáncer de piel mostrada en la prensa condiciona una mayor percepción de riesgo atribuido a otros, la conversación interpersonal fomenta las preocupaciones personales.

De manera especial los individuos ya implicados en la conducta insana perciben el riesgo personal como menor que el riesgo objetivo, por lo que se deja llevar por un "optimismo" irreal (Dillard, McCaul, y Klein, 2006). A modo de ejemplo, los fumadores "optimistas" tienden a pensar que padecer de cáncer de pulmón depende de la herencia genética y que muchos pacientes se curan, pero no identifican el cese de fumar como una vía potencial de reducir el riesgo personal de cáncer (Dillard, et al., 2006).

La falta de la percepción de riesgo se considera una de las formas de resistencia a los mensajes persuasivos más frecuentes entre los receptores, especialmente cuando se trata de comportamientos adictivos. Esta tendencia se basa típicamente en la creencia de que uno es único e invulnerable ante las consecuencias negativas de cierto comportamiento (falta de percepción individual de probabilidad de daño, o percepción de invulnerabilidad). Esto le lleva consecuentemente a la resistencia al mensaje persuasivo porque el individuo 
supone que este no se refiere a él/ella (Moyer-Gusé y Nabi, 2010). La invulnerabilidad percibida representa un "sesgo optimista" independiente del nivel de conocimiento e información sobre una cuestión de salud del que individuo dispone. Debido la invulnerabilidad percibida, los individuos se involucran en comportamientos de riesgo, a pesar de amplio conocimiento que tienen sobre las consecuencias nocivas que estos conllevan (a modo de ejemplo, practican el comportamiento sexual de riesgo, abusan de sustancias lícitas e ilícitas, etc.). Asimismo, los individuos pueden considerar que las consecuencias de una conducta no recomendada no son tan severas como para renunciar a ella, en comparación con el placer o los beneficios que conlleva (percepción de ausencia de severidad).

Las creencias optimistas sobre la salud propia parecen ser especialmente resistentes a las intervenciones mediáticas diseñadas para comunicar riesgo y producir un cambio de conducta. Por este motivo, la investigación en la comunicación de salud se interesa por los factores y las circunstancias decisivas bajo las cuales un mensaje persuasivo alienta la percepción de riesgo. Teniendo en cuenta que la percepción de riesgo en gran medida es un sentimiento, no es de extrañar que las historias y formatos narrativos "vívidos" son más eficaces que la argumentación objetiva de probabilidades estadísticas de riesgo a la hora de fomentar las preocupaciones de salud personal (Chang, 2008; Braverman, 2008; Dillard et al., 2010; Murphy et al., 2013; Wit, Das, y Vet, 2008). En el marco de la investigación que explora el potencial persuasivo del formato narrativo se detectan algunos mecanismos mediadores que explican la eficacia de este formato, tales como el transporte narrativo o la identificación con los persoanjes de la historia. Basil y Brown (1997) encontraron que la identificación con Magic Johnson testificando sobre SIDA predijo la eficacia del mensaje mediático en alentar el riesgo personal de esta enfermedad, además de aumentar la notoriedad del problema y la percepción social. En el contexto del embarazo no deseado, Moyer-Gusé y Nabi (2010) también encuentran apoyo empírico para el efecto positivo de la identificación con los protagonistas de la historia sobre la vulnerabilidad percibida que, a su vez, incrementó las intenciones de practicar el sexo seguro. Un estudio reciente de De Graaf (2014) revela que la similitud entre el receptor y los personajes que protagonizan el mensaje aumenta la percepción de riesgo personal de padecer cáncer de colón. La manera de la que a las personas se les presenta un mensaje de salud, en gran medida condicionan su respuesta y la toma de decisiones en términos de riesgos y beneficios en salud (Slovic et al., 2005: 38).

\section{Conclusiones}

En los últimos años se examinaron diferentes factores que influyen en la percepción pública de riesgo, un parámetro determinante para diseñar la política de salud pública y gestión de riesgos de salud. El gran avance del paradigma psicométrico supone que la percepción de riesgo de las personas no se basa solo en la evaluación del riesgo real, sino también en los sentimientos acerca de la naturaleza del peligro (lo desconocido, incierto, estigmatizado o lo terrorífico que se 
percibe). Entender los factores personales, sociales y las implicaciones de estos procesos afectivos en la percepción de riesgo puede ser crucial para crear buenas estrategias de gestión y comunicación de riesgo en salud. Por tanto, el desafío para los investigadores y comunicadores es aplicar este conocimiento para diseñar estrategias prometedoras en las intervenciones de salud para superar la negación del riesgo personal de los receptores.

\section{Referencias}

Bachman, J. G., Johnston, L. D., O'Malley, P. M., y Humphrey, R. H. (1988). Explaining the recent decline in marijuana use: Differentiating the effects of perceived risks, disapproval, and general lifestyle factors. Journal of Health and Social Behavior, 29, 92-112.

Barrett, L. F., y Salovey, P. (Eds.). (2002). The Wisdom in Feeling. New York: Guilford.

Bouyer, M., Bagdassarian, S., Chaabanne, S., y Mullet, E. (2001). Personality correlates of risk perception. Risk analysis, 21(3), 457-466.

Brady, J. T. (2012). Health risk perceptions across time in the USA. Journal of Risk Research, 15(6), 547-563.

Braverman, J. (2008). Testimonial versus informational persuasive messages: The 
moderating effect of delivery mode and personal involvement. Communication Research, 35, 666-694.

Brewer, N. T., Chapman, G. B., Gibbons, F. X., Gerrard, M., McCaul, K. D., y Weinstein, N. D. (2007). Meta-analysis of the relationship between risk perception and health behavior: the example of vaccination. Health Psychology, 26(2), 136-145.

Chang, C. (2008). Increasing mental health literacy via narrative advertising. Journal of Health Communication, 13, 37-55.

Chauvin, B., Hermand, D., y Mullet, E. (2008). New age beliefs and societal risk perception. Journal of Applied Social Psychology, 38(8), 2056-2071.

De Graaf, A. (2014). The Effectiveness of Adaptation of the Protagonist in Narrative Impact: Similarity Influences Health Beliefs Through Self- Referencing. Human Communication Research, 40(1), 73-90.

De Wit, J. B., Das, E., \& Vet, R. (2008). What works best: objective statistics or a personal testimonial? An assessment of the persuasive effects of different types of message evidence on risk perception. Health Psychology, 27(1), 110-115.

Dillard, A. J., Fagerlin, A., Dal Cin, S., Zikmund-Fisher, B. J., y Ubel, P. A. (2010). Narratives that address affective forecasting errors reduce perceived barriers to colorectal cancer screening. Social science \& medicine, 71(1), 45-52.

Dillard, A. J., Ferrer, R. A., Ubel, P. A., y Fagerlin, A. (2012). Risk perception measures' associations with behavior intentions, affect, and cognition following colon cancer screening messages. Health psychology, 31(1), 106-113.

Dillard, A. J., McCaul, K. D., y Klein, W. M. (2006). Unrealistic optimism in smokers: Implications for smoking myth endorsement and self-protective motivation. Journal of Health Communication, 11(S1), 93-102.

Epstein, S. (1994). Integration of the cognitive and the psychodynamic unconscious. American Psychologist, 49, 709-724.

Fischhoff, B., Slovic, P., Lichtenstein, S., Read, S., y Combs, B. (1978). How safe is safe enough? A psychometric study of attitudes towards technological risks and benefits. Policy sciences, 9(2), 127-152.

Floyd, D. L., Prentice- Dunn, S., y Rogers, R. W. (2000). A meta- analysis of research on protection motivation theory. Journal of applied social psychology, 30(2), 407-429.

Morton, T. A., y Duck, J. M. (2001). Communication and health beliefs mass and interpersonal influences on perceptions of risk to self and others. Communication Research, 28(5), 602-626. 
Moyer- Gusé, E., y Nabi, R. L. (2010). Explaining the effects of narrative in an entertainment television program: Overcoming resistance to persuasion. Human Communication Research, 36(1), 26-52.

Murphy, S. T., Frank, L. B., Chatterjee, J. S., y Baezconde- Garbanati, L. (2013). Narrative versus nonnarrative: The role of identification, transportation, and emotion in reducing health disparities. Journal of Communication, 63, 116-137.

Rogers, R. W. (1975). A protection motivation theory of fear appeals and attitude change. Journal of Psychology, 91(1), 93-114.

Rogers, R. W. (1983). Cognitive and physiological processes in fear appeals and attitude change: a revised theory of protection motivation. En J. T. Cacioppo y R. E. Petty (Eds.), Social psychophysiology. A sourcebook (pp. 153-176). Nueva York: The Guilford Press.

Rosenstock, I. M. (1974). Historical origins of the health belief model. Health Education \& Behavior, 2(4), 328-335.

Sloman, S. A. (1996). The empirical case for two systems of reasoning. Psychological Bulletin, 119(1), 3-22.

Slovic, P. (1987). Perception of risk. Science, 236(4799), 280-285.

Slovic, P., Finucane, M. L., Peters, E., y MacGregor, D. G. (2004). Risk as analysis and risk as feelings: some thoughts about affect, reason, risk, and rationality. Risk Analysis, 24(2), 311-322.

Slovic, P., Fischhoff, B., y Lichtenstein, S. (1982). Why study risk perception?. Risk analysis, 2(2), 83-93.

Slovic, P., Peters, E., Finucane, M. L., y MacGregor, D. G. (2005). Affect, risk, and decision making. Health psychology, 24(4S), S35-S40.

Slovic, P., y Peters, E. (2006). Risk perception and affect. Current directions in psychological science, 15(6), 322-325.

Slovic, P., y Weber, E. U. (abril, 2002). Perception of risk posed by extreme events. Ponencia presentada en el congreso "Risk Management strategies in an Uncertain World," Palisades, New York. Recuperado de https://www.Ideo.columbia.edu/chrr/documents/meetings/roundtable/white papers/sI ovic wp.pdf

Weber, E. U. (2001b). Decision and choice: Risk, empirical studies. In N. J. Smelser y P. B. Baltes (Eds.), international encyclopedia of the social and behavioral sciences (pp. 13347- 13351). Oxford, UK: Elsevier Science Limited. 
Weinstein, N. D., Kwitel, A., McCaul, K. D., Magnan, R. E., Gerrard, M., y Gibbons, F. X. (2007). Risk perceptions: assessment and relationship to influenza vaccination. Health Psychology, 26(2), 146-151.

Witte, K. (1992). Putting the fear back into fear appeals: The extended parallel process model. Communications Monographs, 59(4), 329-349.

Zajonc, R. B. (1980). Feeling and thinking: Preferences need no inferences. American Psychologist, 35, 151-175. 